\title{
Evaluation of different immunoassays for the quantitative detection of Epstein-Barr virus capsid antigen immunoglobulin A using a candidate reference
}

Hao Chen

Qiaohua Zhong

PEOPLE HOSPITAL OF JIEYANG

Xiaobin Wu

Guangdong Hospital of Traditional Chinese Medicine

Yanling Ding

LIUZHOU Maternity and Child Health Care Hospital

Qi Chen

PEOPLE Hospital OF JIEYANG

Ning Xue

Henan Cancer Hospital

Weizhong Chen

CHAOZHOU PEOPLE'S HOSPITAL

shan xing

SYSUCC

yiwei xu ( $\square$ yiwei512@126.com )

Shantou University Medical College Cancer Hospital

Research article

Keywords: Epstein-Barr virus, Nasopharyngeal carcinoma, virus capsid antigen, reference, enzyme-linked immunosorbent assay (ELISA)

Posted Date: July 31st, 2019

DOI: https://doi.org/10.21203/rs.2.12248/v1

License: (9) This work is licensed under a Creative Commons Attribution 4.0 International License.

Read Full License 


\section{Abstract}

Background As Epstein-Barr capsid antigen (VCA) immunoglobulin A (IgA) detection is widely used for nasopharyngeal carcinoma (NPC), many immunoassays are available for VCA- IgA detection. Methods We evaluated the performance of different immunoassays for the quantitative detection of VCA-IgA. Twenty NPC patients were detected VCA-IgA by four ELISA kits and twochemiluminescent immunoassays(CLIA) kits using the reference as a calibration curve. The performance of six kits were evaluated, and the quantitative results were compared. Results Our results shown the reference has good linearity in different kits. Without reference, the difference of total CV (from 3.98\% to 43.11\%) and Withinrun $\mathrm{CV}$ (from $2.47 \%$ to 19.66\%) was large in 6 kits. The positive and negative coincidence rate between 6 kits and IFA for NPC diagnosis was 75\% overall agreement, but there was a difference among the six kits ranged from $55 \%-90 \%$. Concentration of samplesdivided into three categories was shown significant difference in certain subgroup of 6 kits. Almost all assays gave a correlation coefficient lower than 0.80 , except for Antu and Beier kit and Tarcine kit and New Industries kit. Conclusions The reference serum may be used as a reference standard and better compared results from different methods/laboratories.Despite the use of our reference, the quantitative results of each kit are still quite different.We should try to unify the diversity between different antigens to promote the development of VCA-related research in the further.

\section{Background}

South China is a highly endemic area of Nasopharyngeal carcinoma (NPC). The ASIRW(age-standardized incidence rates of world)in South China $(9.69 / 100,000)$ was 3.4 times higher than that in Southwest China $(2.85 / 100,000)$, in which the incidence is the second highest. [1, 2]Epstein-Barr virus (EBV) infection is one of the most important factor for NPC onset.[3]EBV associated antibodies, such as virus capsid antigen (VCA-IgA) and Epstein-Barr virus nuclear antigen 1 (EBNA1-IgA), exert an important role in the screening and diagnosis of NPC.[4]VCA-IgA is one of the most widely antibody markers used for assisting in monitoring efficacy of NPC and screening NPC.[5][6-8] It was usually measured by indirect immunofluorescence(IFA)[9] or enzyme-linked immunosorbent assay (ELISA)[10]. IFA was used as the "gold standard" for detection of VCA-Ig A antibody. In virtue of enzyme-labeled antibodies instead of fluorescent antibodies, IEA did not need a fluorescence microscope to interpret the results which the semiquantitative results were reported by titers was widely used in southern China.[2, 3].

The titer of VCA-IgA was closely related to the longterm curative effect and prognosis of NPC.[11, 12] Low titer has better efficacy and prognosis. Moreover, ascending EBV antibodies titers are strongly associated with an increased risk for NPC. $[13,14]$ Although, the titer detected by traditional IEA or IFA has considerable prognostic value, it is not suitable for mass NPC screening because of the need for manual interpretation. ELISA-based assays have similar detection sensitivity and specificity to IFA, $[4,15]$ and have other advantages. ELISA-based assays are easier to be standardized, with greater false tolerance to interference, and are also more labor-saving when analyzing a large number of specimens. 
Chemiluminescent immunoassay (CLIA) has advantages similar to ELISAhave been developed in China, but no literature has been reported yet.

In summary, ELISA and CLIA are convenient and automated. But the performance and standardization of ELISAs and CLIAs used different peptide segmentsof VCA are still a matter of debate and concern. Inaddition, while new methodologies and new platforms (automated vs.manual, ELISA vs.CLIA) for the detection of VCA-IgA have become available, proper interassay simultaneous comparisons have not been carried out. One important topic of discussion is whether to use nature reference preparations to calibrate these assays for quantitative testing. And the proper cross-validation and evaluation of the available for different methods and brands of kits are lacking.

But so far, reference standard for VCA-IgA has not yet been determined by the World Health Organization (WHO) International Laboratory and Collaborating Centers for Biological Standards. Most commercial ELISA kits only generate either negative or positive results.[16]Although our previous studies have confirmed that the rOD value of ELISA shows a good correlation with the titer from IEA. [2]At present, VCAIgA detected by ELISA is unable to be used as a prognostic factor for NPC as IFA. As VCA-IgA ELISA is widely used in clinical laboratories, calibrator is in urgent need. Recently, at the request of the Guangdong Provincial Anticancer Association (http://www.gdaca.org.cn/), Southern China Tumor Markers Standardization Alliance (Guangzhou, guangdong, P. R.China) was given a potential reference sample from Zhong He (Guangzhou, China) and Sun Yat-sen University Cancer Center(SYSUCC, Guangzhou, China), obtained from three patients with NPC and mixed with serum samples from healthy subjects for dilution purposes. This will be made available as the first international VCA-IgA reference reagent if it fulfils certain criteria for such use. We have performed a collaborative study in six clinical laboratories to evaluate different immunoassays in the quantitativedetection ofVCA-IgAand verify whether improve comparability of the results usingthisreference.

\section{Method}

\section{Preparation of the reference serum}

The VCA-IgA reference sample was prepared using serum from three strongly VCA-IgA positive NPC patients who were histologically confirmed by biopsy. Each patient took $20 \mathrm{ml}$ of blood and added $\mathrm{CaCl}_{2}$ to a final concentration of $18 \mathrm{mmol} / \mathrm{L}$. Subsequently, the solution was incubated for $2 \mathrm{~h}$ at $37^{\circ} \mathrm{C}$ and the insoluble material was removedby centrifugation (30 min at 10000rpm). Finally, the supernatant was filtered with a $0.2 \mu \mathrm{m}$ pore size nitrocellulose fiter.

The VCA-IgA reference material was prepared by diluting the NPC serum by testing a series of dilutions in the ELISA assay from Euroimmun AG (Lubeck,Germany) and chemiluminescent immunoassay(CLIA) from New Industries (Shenzhen, China). The concentration closed to upper limit of detection method was identified as the best dilution concentration. And this optimum concentration was assigned to a target value of $16000 \mathrm{U} / \mathrm{ml}$. Serum samples obtained from healthy blood donors were mixed to a pool as a 
dilutions. The donors' serum samples were individually tested in the Euroimmun IFA assay and were all found to be VCA-IgA negative. All serum samples were also found to be negative for anti-HIV $1+2, \mathrm{HbsAg}$ and anti-HCV(Hepatitis C Virus).

The bulk material was produced by diluting the VCA-IgA-positive NPC serum in pooled serum from 50 blood donors, using the optimal dilution factor as determined above. Finally, 1 liter bulk reference serum of VCA-IgA was prepared. Subsequently, the bulk prepared reference serum was divided into $0.2 \mathrm{ml}$ portions and preserved in $-80^{\circ} \mathrm{C}$ refrigerator.

\section{Patients}

Twenty patients with NPC were confirmed by histologically biopsy and further tests, including head and neck MRI and chest X-rays. Negativity and low and medium/high positivity was defined as result of VCAIgA titer detected by VCA-IgA Euroimmun IFA. Seven were negative(titer<1:20), seven were low

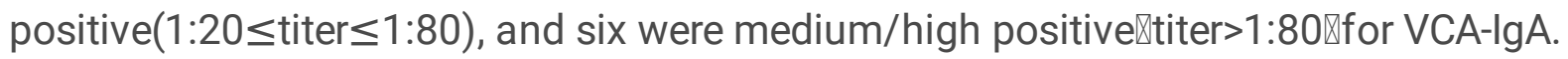

\section{VCA-IgA measurements}

For the evaluation of VCA-IgA levels, we used 6 commercial kits to analyze the reference and sera from 20 patients with NPC. Four immuno-enzymatic (ELISA) commercial kits produced by the following manufacturers: Euroimmun AG (Lubeck,Germany), Tarcine BioMed (Beijing, China), Antubio (Zhengzhou, China), Beier (Beijing, China). And 2 chemiluminescent immunoassay (CLIA) kits produced by the following manufacturers and tested by matching detection systems: New Industries Biomedical Engineering (Maglumi 4000 Plus System, Shenzhen, China) and YHLO Biotech (iFlash 3000 CLIA System,Shenzhen, China). Antigen peptides, substrate, incubation time, volume and dilution of serum, type of conjugate, enzymatic substrate and cut-off level of six kits are not exactly the same. (table 1)

VCA-IgA titer of 20 patients were also tested by Immunofluorescence assay (IFA) as the gold standard method.

\section{Linearity, precisionand comparability}

The reference serum was serially prediluted from 1:1 to 1:64 by dilutions in respective kits. The linearity of the measurements was calculated by means of linear regression statistical analysis(slope value with $95 \% \mathrm{Cl}$, adjusted $\mathrm{R}^{2}$ ) using the original reference and seven predilutions of the reference serum were further diluted according to the manufacturer's protocol, with each of the 6 commercial methods (table 1).

The precision was evaluated according to the EP15-A2 protocol of the Clinical and Laboratory Standards Institute (CLSI).[17] The original reference and 1:16 predilution were analyzed three times per day during a period of five consecutive workdays( $n=15$ per level). Precision was evaluated as the coefficient of variation (CV), which was calculated from the data series mean and standard deviation. 
To evaluate the efficacy of harmonisation of antibody levels,sera from 20 NPC patients with different VCA-IgA concentrations (13 positive and seven negative, as measured in the IFA were analyzed with 6 commercial kits. The VCA-lgA concentrations were calculated by calibration curve obtained by the original and seven dilutions of the reference serum. A concentration value of 16000 units $(\mathrm{U} / \mathrm{ml})$ was arbitrarily assigned to the original reference serum.

\section{Statistical analysis}

Scatter diagram and Linear regression analysis were performed by MedCalc version 11.4.2.0. The VCAIgA concentration of each patient by 6 methods divided into three sample categories was compared via randomized block design ANOVA. Then, LSD test were used to make multiple comparisons between the groups. Quantitative results were correlated by the non-parametricSpearman test. The statistical analyseswere performed using IBM SPSS Statistics version19.0 (IBM Corp.). Statistical signifcance was considered present at $p<0.05$.

\section{Results}

\section{Linearity}

Linear regression analysis was performed to evaluate the linearity of the quantitative data. Graphs and statistical values(slope with $95 \% \mathrm{Cl}$, adjusted $\mathrm{R}^{2}$ ) are shown in figure 1 and table $\mathrm{S} 1$. Coefficients of regression as a measure of linearity of the diluted reference serum tested with each one of the 6 commercial methods ranged from 0.00007181 to 11.276 (table S1). Except for Tarcine $\left(R^{2}>0.9367\right)$, regression analysis for the other 5 commercial kits had achieved a good correlation coefficient $\left(R^{2}>0.96\right)$ over the entire range tested. (figure 1 ,table $\mathrm{S} 1$ )

To verify whether linearity was present even at low and high antibody concentrations, we diluted one low and one high VCA-IgA serum in the same way in which we had diluted the reference sample (predilutions from 1:0 to 1:64 followed by the dilution requested in each assay). The results showed that linearity was maintained even when low antibody concentrations, but did not maintained in upper high antibody concentrations(Figure S1).

\section{Precision}

The VCA-IgA concentrations of Euroimmun and Tarcine kits were calculated by one calibrator curve provided in each kit. The VCA-IgA concentrations of New Industries and YHLO kits were calculated by the calibration curve provided in each kit. Since the Antu and Beier ELISA kits do not have a self-made calibrator, precision was calculated by OD value of each detection when all the negative and positive controls met manufacturer requirements. In addition to Beier ELISA kit, other 5 kits have excellent withinrun $\mathrm{CVs}(\mathrm{CV}<8 \%)$ at the low and high VCA-IgA levels. Except for Antu and Beier ELISA kits, other 4 kits also have good total $\mathrm{CVs}(\mathrm{CV}<10 \%)$. (table2) 


\section{Comparability}

Table 2 shows the VCA-IgA values for the highest concentration of the reference serum obtained by the 6 commercial methods in each kit, the cut-off values proposed by each manufacturer, and their ratio. The VCA-IgA concentrations varied from 1.4 arbitrary units given by the Beier kit to 13 arbitrary units given by the Euroimmun kit, showing a very wide dispersion of the quantitative data.

The VCA-IgA concentration of each patient by different methods were shown in line chart. (figure $2 A, B, C$ ) All three sample categories (negative and low and medium/high positive) detected by 6 kits when results were calibrated by the reference material displayed significant difference beteen certain of six methodsaccording to LSD test $(P<0.05)$. ( figure 2D,E,F) The value of New Industries and other kits were a little different. New Industrieskit was different from other methods in medium/high positive categories by LSD test $(\mathrm{P}<0.05)$. (figure $2 \mathrm{~F})$ It was also different from Euroimmun, Beier and Antu kits in negative category. (figure 2D) And it was also different from Euroimmun kit in low positive category. (figure 2E)

\section{Correlation between six assays}

Positive rates of each method were analyzed in 20 NPC patients.(Table 3) Euroimmun ELISA showed the highest positive rate in seven methods. The positive and negative coincidence rates between six method and gold standard method (IFA) are the same, but the differences between six method are great(ranged from $55 \%-90 \%$ ). (Table 3 ) And the positive and negative coincidence rates of the four ELISA methods(Euroimmun and Beier and Tarcine and Antubio) were more than 70\%.In addition, Beier and New Industries had the highest positive-negative coincidence rate, reaching $90 \%$.

Table 4show the Spearman correlation coefficientsbetween the different assays. Almost all assays gave a correlation coefficient lower than 0.80.Although quantitative results among different methods are not exactly the same, a good correlation of was observed between Antu and Beier ELISA kit(0.9857),, and between Tarcine ELISA kit and New Industries CLIA kit (0.9459)..

\section{Discussion}

As the VCA-IgA was in widespread use in clinical laboratories as a major maker for NPC, three methods include IFA, ELISA and CLIA for VCA-IgA antibody detection are available. Numerous manufacturers used different peptides as VCA antigens with different assays has greatly contributed to the dissemination of the NPC screening in clinical laboratories, but the need for comparison of the results obtained with different kits and the urgent need for standardisation of the assay have become a priority. The main objective of this study was to evaluate and compare the performance of different commercial ELISAs and other new immunoassays for the quantitative detection of VCA-IgA. Another important objective was to evaluate the candidate reference serum by six different kits in six laboratories. To this end, a freeze reference sample was prepared by Zhong He (Guangzhou Zhong He Biotechnology Co., Ltd.) and proposed as a candidate reference serum for VCA-IgA detection to the Southern China Tumor Markers Standardization Alliance. This reference was provisionally tested and proved to be consistent with the 
requirements before and after freezing and thawing. (date not shown) The reference serum showed positive results in all the commercial VCA-IgA kits used. Linear analysis by reference standard curve obtained in all 6 kits showed that the reference sample can be used as a calibrator in assays using different antigenic substrates or different assays. Residual differences may be due to different assay reagents/procedure (dilution buffer, antigenic peptides, sample dilution, etc). Since the linearity of the method cannot be maintained at a higher concentration and the linearity $\left(R^{2}\right)$ does not reach 0.99 , it is recommended to use multi-point curves for each kit in the future.

Owing to the same reason of without reference, the precision of each kit is quite different. At present, the VCA-IgA kits can be classified into three categories according to the condition of the kit calibrator. First category, such as Beier and Antu VCA-IgA ELISA kits, they do not have any calibration material, but only negative and positive quality controls. The results were accepted when the negative and positive controls were acceptable within a certain range. Therefore, the results within the batch (Within-run CV) may not be very different, but the results of each batch(Total CV) are quite different. There is no comparison between the quantitative results of different test batches. The second category, such as Euroimmun and Tarcine VCA-IgA ELISA kits, the kit has a critical calibrator with its own. They have satisfactory within-run CV and total CV. But the disadvantage of single point calibrator is that rODs(OD/cut-off OD) is difficult to accurately express all values because of imperfect linearity $(R 2=0.9779$ and $R 2=0.9367)$. The third category, such as New Industries and YHLO VCA-IgA CLIA kits, they own calibration curves and provide quantitative results using their own units. Because of the advantages of methodology and matched testing system, they have better Within-run CV and Total CV.

Up to now, there is no systematic evaluation study on the quantitative and standardized research of all VCA-IgA methods.Previous studies on VCA-IgA detection generally used ELISA and IFA,[4] CLIA only related to VCA IgG or IgM antibody tested. In this study, we first included CLIA in VCA-IgA research.[18, 19]

The coincidence rate between the six methods and the gold standard method (IFA) was 75\%. However, the coincidence rate of positive and negative between the six methods was quite different. (Table 3 ) Actually, the results obtained by different methods and expressed in arbitrary units differed so much that it was quite difficult to compare data from different studies and to understand whether VCA-IgA antibody levels could be used for monitoring therapy and prognostic purposes. Many studies have confirmed that VCA-IgA antibody level changes can be used for NPC prognosis and screening.[13, 20-22] Pretreatment serum EBV-VCA/IgA titer may be used as an independent prognostic marker of NPC.[11] Liuet al.'s study found that the geometric mean titer of anti-EBV/VCA IgA antibodies before and after radiotherapy was a significant difference.[23] People with ascending VCA-IgA antibody titers tended to have higher risks and shorter time to develop NPC, compared to those with a descending pattern.[13] For both the ELISA and CLIA, there was good agreement with IFA. Concordance between 6 kits and IFA for primary NPC diagnosis was $75 \%$ overall agreement.(table 3) This result was similar to Karray's research which observed $81-91 \%$ overall agreement between ELISA(IgA anti-VCA-p18) and IFA for primary NPC diagnosis. They also found declining reactivity in patients in remission and increasing reactivity in patients with persistent disease or relapseduring follow-up monitoring both in ELISA and IFA detected antibody.[15] Our previous study also 
found an excellent correlation and a high degree of concordance between IgA titers(IEA) and rOD levels (ELISA),[2] However, Yao et al.'s study did not confirm the role of VCA-IgA as prognostic biomarkers among patients with NPC and undetectable EBV DNA. [24] A possible explanation for these contradictory results may be that the previous studies supporting the positive results use IFA, however this study uses ELISA囚Beier kit囚. In the absence of calibrators, inter-assay differences in ELISA may affect outcome analysis, which is documented in the limitations of this article [24] and is confirmed in our study $\otimes T$ Total CV $=43.11 \%$. This confirms the importance of the reference in VCA-IgA ELISA detection again.

Through reference, we may connect each different methodand analyze the results which were not comparable before. We observed that the same patient has similar results with some different methods, or that the values are different but the trends are the same with different methods. $₫$ figure $2 \bigotimes$ The results of twenty patients tested by the kits calibrated on the reference serum divided into three sample categories was shown certain significant difference in 6 methods compared via randomized block design ANOVA and further analysis according to LSD test. (figure 2) New Industries kit is different from other methods in medium/high positive categories by LSD test $(P<0.05)$. Moreover, New Industries and Euroimmun were different in the three groups.

Although there are still differences in quantitative results among different methods, the improvement of this study is obvious. The reference material can reduce the difference between different batches with the same kit. Furthermore, the quantitative results by reference standard curves are more accurate than the semi-quantitative results of IFA for monitoring efficacy and risk. IFA/IEA as the "gold standard" for detection of IgA to VCA, are laborious techniques, since they cannot be sufficiently automated to achieve a good level of output, and there is a certain degree of subjectivity in interpreting results. ELISA can achieve quantitative detection through calibrationcurve,and is easy to automate and detect a large scale of samples quickly. Finally, through reference materials, we may compare the results of different manufacturers' kits. A good correlation(correlations coefficient $=0.9857$ ) was observed between Antu and Beier ELISA kits, which results calculated by reference standard curves. And the same correlation (correlations coefficient $=0.9459$ ) was observed between Tarcine and New Industries.

Although different kits calibrated by the reference serum displayed a certain consistency of VCA-IgA antibody results, there are still some shortcomings in comparing the results of different methods. For example, the quantification value of a particular patient was quick differences between some methods. As regards measurements of IgA, all assays used the p18 peptide, except Euroimmun, which used gp125. In addition, the other three assays (Antu,New Industries,YHLO) which use the peptide p18 also contains other peptides. The results depend on the use of a range of different antigens, which can produce different serological responses. Although on the use of the same antigen, otherfactors, such as the different dilutions used for analysismaybe differences.[25]These differences may also lead to the different outcome of therapeutic and prognostic studies using different VCA-IgA kits.

\section{Conclusions}


In summary, the quantitative results of each kit are quite different at present. The use of the reference serum may a certain reduce the differences between different kits and achieve a comparison between different kits. In subsequent studies, we should focus on the diversity between different antigens and try to unify them to promote the further development of VCA-related research.

\section{Abbreviations}

nasopharyngeal carcinoma (NPC) 『virus capsid antigen (VCA)『immunoglobulin A ( $\lg A) \rrbracket$ chemiluminescent immunoassays (CLIA)『Epstein-Barr virus nuclear antigen 1 (EBNA1-IgA) 『indirect immunofluorescence(IFA)『enzyme-linked immunosorbent assay (ELISA)『Epstein-Barr virus (EBV)『early antigen (EA)『immunoenzymatic assay (IEA)『World Health Organization (WHO)『age-standardized

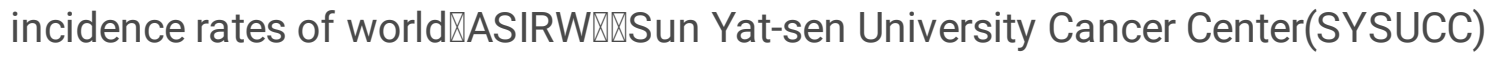

\section{Declarations}

\section{Ethics approval and consent to participate}

The study follows the principles of the Declaration of Helsinki. This study was approved by the Institutional Review Board of Sun Yat-sen University Cancer Center. The authenticity of this article has been validated by uploading the key raw data onto the Research Data Deposit public platform (www.researchdata.org.cn), with the approval RDD number as RDDA2019001095

\section{Consent for publication}

All patients provided written-informed consent for the collection and publication of their medical information at the first visit to our center, which was filed in their medical records, and the ethics committees approvedthis consent procedure.

\section{Availability of data and materials}

The datasets used and/or analysed during the current study are available from the corresponding author on reasonable request.

\section{Conflict of Interests: NONE}

\section{Funding}

This work was supported by the Natural Science Foundation of Guangdong

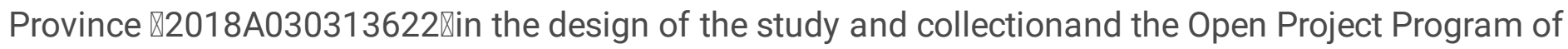
the State Key Laboratory of Proteomics (SKLP0201703)in writing the manuscript and the National Natural Science Foundation of China (no. 31600632) in analysis. 
YWX and SX conceived and designed the experiments. HC and QC and XBW and NX and WZC and YLD performed theexperiments: $\mathrm{HC}$ and $\mathrm{QHZ}$ analyzed the data. $\mathrm{HC}$ and $\mathrm{YWX}$ wrote the paper.All authors read and approved the final manuscript.

\section{Acknowledgments}

We thank the following: all members of the Guangdong Provincial Anticancer Association and Southern China Tumor Markers Standardization Alliance for their support and, in particular, the WL Liu and Hao Chen, for their initiative and efforts in preparing the reference sample.

\section{Additional files}

Additional file 1:Table S1. A summary of linear regression studies

Additional file 2: Figure S1. The regression curves obtained by diluted one more low and high VCA-IgA serum concentrations with each of the 6 commercial methods. Values of the ordinate are givenin the units used by the assay kit, while the abscissa shows the arbitrary units of the reference sample.

\section{References}

[1] Chen W, Sun K, Zheng R, Zeng H, Zhang S, Xia C, et al. Cancer incidence and mortality in China, 2014. Chinese journal of cancer research $=$ Chung-kuo yen cheng yen chiu. 2018;30:1-12.

[2] Chen H, Chi P, Wang W, Li L, Luo Y, Fu J, et al. Evaluation of a semi-quantitative ELISA for IgA antibody against Epstein-Barr virus capsid antigen in the serological diagnosis of nasopharyngeal carcinoma. International journal of infectious diseases: IJID: official publication of the International Society for Infectious Diseases. 2014;25:110-5.

[3] Chen H, Chen S, Lu J, Wang X, Li J, Li L, et al. Multiparametric Detection of Antibodies against Different EBV Antigens to Predict Risk for Nasopharyngeal Carcinoma in a High-Risk Population of China. Cancer Prev Res (Phila). 2017;10:542-50.

[4] Liu Y, Huang Q, Liu W, Liu Q, Jia W, Chang E, et al. Establishment of VCA and EBNA1 IgA-based combination by enzyme-linked immunosorbent assay as preferred screening method for nasopharyngeal carcinoma: a two-stage design with a preliminary performance study and a mass screening in southern China. International journal of cancer. 2012;131:406-16.

[5] Henle G, Henle W. Epstein-Barr virus-specific IgA serum antibodies as an outstanding feature of nasopharyngeal carcinoma. International journal of cancer. 1976;17:1-7.

[6] Chien YC, Chen JY, Liu MY, Yang HI, Hsu MM, Chen CJ, et al. Serologic markers of Epstein-Barr virus infection and nasopharyngeal carcinoma in Taiwanese men. The New England journal of medicine. 2001;345:1877-82. 
[7] Zeng Y, Zhang LG, Li HY, Jan MG, Zhang Q, Wu YC, et al. Serological mass survey for early detection of nasopharyngeal carcinoma in Wuzhou City, China. International journal of cancer. 1982;29:139-41.

[8] Zong YS, Sham JS, Ng MH, Ou XT, Guo YQ, Zheng SA, et al. Immunoglobulin A against viral capsid antigen of Epstein-Barr virus and indirect mirror examination of the nasopharynx in the detection of asymptomatic nasopharyngeal carcinoma. Cancer. 1992;69:3-7.

[9] Chen Y, Xin X, Cui Z, Zheng Y, Guo J, Lin Y, et al. Diagnostic Value of Serum Epstein-Barr Virus Capsid Antigen-IgA for Nasopharyngeal Carcinoma: a Meta-Analysis Based on 21 Studies. Clinical laboratory. 2016;62:1155-66.

[10] Li S, Deng Y, Li X, Chen QP, Liao XC, Qin X. Diagnostic value of Epstein-Barr virus capsid antigen-IgA in nasopharyngeal carcinoma: a meta-analysis. Chinese medical journal. 2010;123:1201-5.

[11] Ling W, Cao SM, Huang QH, Li YH, Deng MQ. Prognostic implication of pretreatment titer of serum immunoglobulin A against Epstein-Barr virus capsid antigen in nasopharyngeal carcinoma patients in Sihui, Guangdong. Ai zheng $=$ Aizheng $=$ Chinese journal of cancer. 2009;28:57-9.

[12] Zeng Y. Seroepidemiological studies on nasopharyngeal carcinoma in China. Advances in cancer research. 1985;44:121-38.

[13] Cao SM, Liu Z, Jia WH, Huang QH, Liu Q, Guo X, et al. Fluctuations of epstein-barr virus serological antibodies and risk for nasopharyngeal carcinoma: a prospective screening study with a 20-year followup. PloS one. 2011;6:e19100.

[14] Du JL, Chen SH, Huang QH, Xie SH, Ye YF, Gao R, et al. Subtype distribution and long-term titer fluctuation patterns of serum anti-Epstein-Barr virus antibodies in a non-nasopharyngeal carcinoma population from an endemic area in South China: a cohort study. Chinese journal of cancer. 2016;35:78.

[15] Karray H, Ayadi W, Fki L, Hammami A, Daoud J, Drira MM, et al. Comparison of three different serological techniques for primary diagnosis and monitoring of nasopharyngeal carcinoma in two age groups from Tunisia. Journal of medical virology. 2005;75:593-602.

[16] Gao R, Wang L, Liu Q, Zhang LF, Ye YF, Xie SH, et al. Evaluation of seven recombinant VCA-IgA ELISA kits for the diagnosis of nasopharyngeal carcinoma in China: a case-control trial. BMJ open. 2017;7:e013211.

[17] Wu X, Chao Y, Wan Z, Wang Y, Ma Y, Ke P, et al. A comparative evaluation of the analytical performances of Capillarys 2 Flex Piercing, Tosoh HLC-723 G8, Premier Hb9210, and Roche Cobas c501 Tina-quant Gen 2 analyzers for HbA1c determination. Biochemia medica. 2016;26:353-64.

[18] Grandjean Lapierre S, Vallieres E, Rabaamad L, Labrecque M, Chartrand C, Renaud C. Evaluation of the abbot Architect() epstein-barr virus viral capsid antigen IgM, viral capsid antigen IgG and nuclear 
antigen IgG assays in a pediatric and adult population. Journal of clinical virology: the official publication of the Pan American Society for Clinical Virology. 2016;81:1-5.

[19] Francois C, Segard C, Bouvier M, Stefanski M, Pannier C, Zawadzki P, et al. Comparison of Abbott Architect((R)), Siemens Immulite((R)), and Diasorin Liaison((R)) for determination of Epstein-Barr virus serological diagnosis. Diagnostic microbiology and infectious disease. 2018;90:96-101.

[20] de-Vathaire F, Sancho-Garnier H, de-The H, Pieddeloup C, Schwaab G, Ho JH, et al. Prognostic value of EBV markers in the clinical management of nasopharyngeal carcinoma (NPC): a multicenter follow-up study. International journal of cancer. 1988;42:176-81.

[21] Lynn TC, Tu SM, Kawamura A, Jr. Long-term follow-up of IgG and IgA antibodies against viral capsid antigens of Epstein-Barr virus in nasopharyngeal carcinoma. The Journal of laryngology and otology. 1985;99:567-72.

[22] Ji MF, Wang DK, Yu YL, Guo YQ, Liang JS, Cheng WM, et al. Sustained elevation of Epstein-Barr virus antibody levels preceding clinical onset of nasopharyngeal carcinoma. British journal of cancer. 2007;96:623-30.

[23] Liu MT, Yeh CY. Prognostic value of anti-Epstein-Barr virus antibodies in nasopharyngeal carcinoma (NPC). Radiation medicine. 1998;16:113-7.

[24] Yao JJ, Lin L, Jin YN, Wang SY, Zhang WJ, Zhang F, et al. Prognostic value of serum Epstein-Barr virus antibodies in patients with nasopharyngeal carcinoma and undetectable pretreatment Epstein-Barr virus DNA. Cancer science. 2017;108:1640-7.

[25] de Ory F, Guisasola ME, Sanz JC, Garcia-Bermejo I. Evaluation of four commercial systems for the diagnosis of Epstein-Barr virus primary infections. Clinical and vaccine immunology: CVI. 2011;18:444-8.

\section{Tables}

Table 1. Characteristics of the 7 commercial kits for detecting VCA-IgA antibodies 


\begin{tabular}{|c|c|c|c|c|c|}
\hline facturer & Method & Antigen & $\begin{array}{c}\text { Chromogenic / luminescence } \\
\text { reaction system }\end{array}$ & $\begin{array}{l}\text { Sample } \\
\text { dilution }\end{array}$ & $\begin{array}{c}\text { Testing } \\
\text { laboratory }\end{array}$ \\
\hline \multirow[t]{2}{*}{ simmun } & IFA & P3HR1 & FITC & $1: 10$ & SYSUCC \\
\hline & ELISA & gp125 & $\mathrm{HRP} / \mathrm{TMB}$ & $1: 100$ & SAHGUCM \\
\hline $\mathrm{r}$ & ELISA & $\mathrm{p} 18$ & $\mathrm{HRP} / \mathrm{TMB}$ & $1: 10$ & CHSUMC \\
\hline ine & ELISA & $\mathrm{p} 18$ & HRP/TMB & $1: 10$ & $\mathrm{PHJ}$ \\
\hline l & ELISA & gp125,p18 & HRP/TMB & $1: 10$ & ATHZU \\
\hline Istries & CLIA & p18,p23 & ABEI & $1: 10$ & SYSUCC \\
\hline $\mathrm{O}$ & CLIA & $\mathrm{p} 18, \mathrm{p} 23$ & Acridinium ester/ H2O2 & $1: 10$ & $\mathrm{LMCHCH}$ \\
\hline
\end{tabular}

TMB, tetramethyl benzidine; ABEI, N-(4-Aminobutyl)-N-ethylisoluminol; SYSUCC, Sun Yatsen University Cancer Center; SAHGUCM, Second Affiliated Hospital of Guangzhou University of Chinese Medicine; CHSUMC, The cancer hospital of shantou University Medical college; PHJ, People's Hospital of Jieyang; ATHZU, Affiliated Tumor Hospital of Zhengzhou University; LMCHCH, Liuzhou Maternity and Child Health Care Hospital 
Table 2 Levels of VCA-IgA antibodies detected in the VCA-IgA antibody reference serum by the 6 commercial methods.

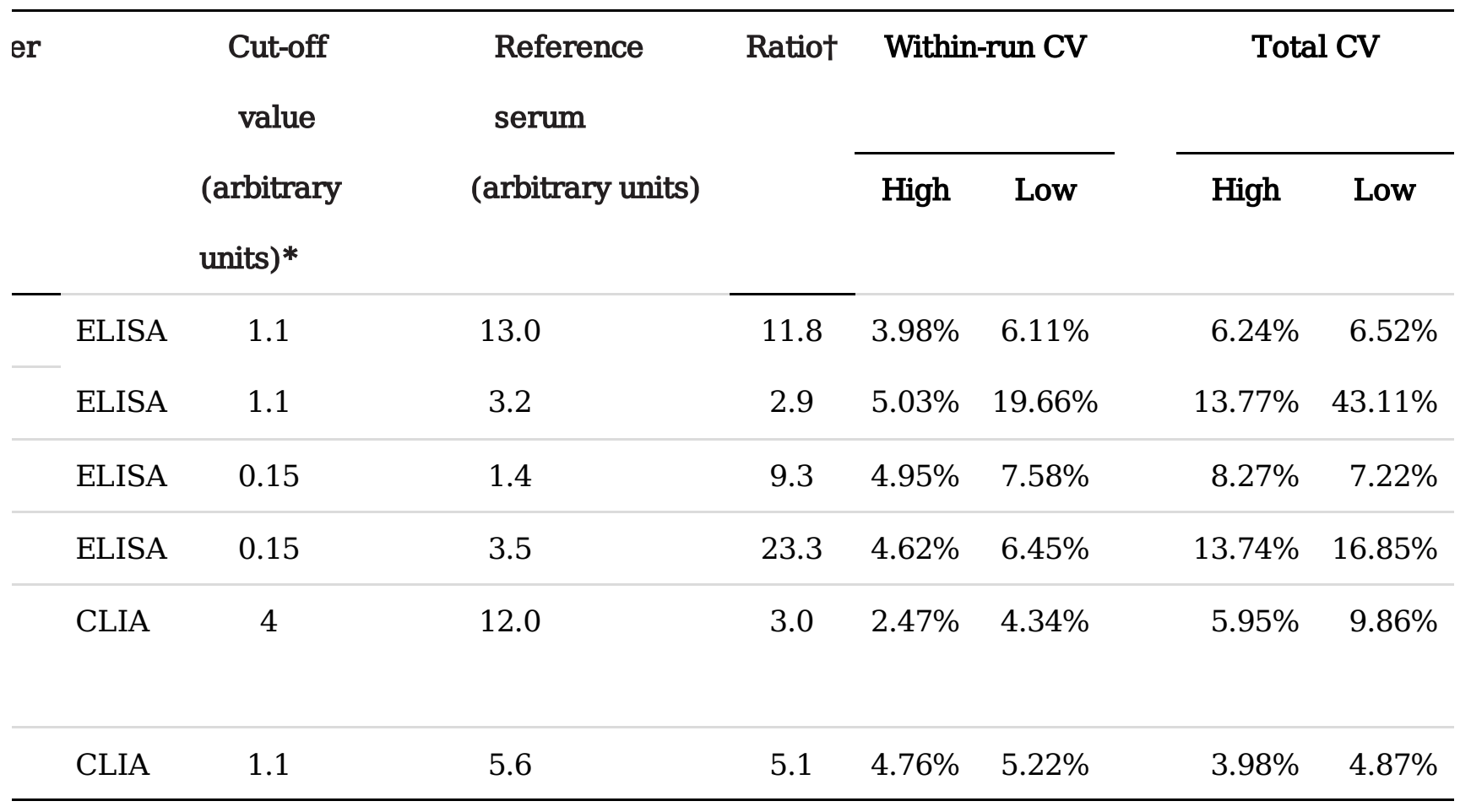

*VCA-IgA antibody levels detected in the reference serum at the highest concentration tested in each kit.

†Ratio was calculated as reference serum arbitrary units divided by manufacturer cutoff value for each kit.

**Precision was calculated by OD value of each test when all the negative and positive controls met manufacturer requirements 
Table 3. Performance and coincidence rate of the different assays

\begin{tabular}{|c|c|c|c|c|c|c|c|}
\hline & \multirow{2}{*}{$\begin{array}{l}\text { Positive } \\
\text { rate(\%) }\end{array}$} & \multicolumn{6}{|c|}{ Coincidence rate of positive and negative $(\%)^{b}$} \\
\hline & & Beier & Tarcine & Antubio & $\begin{array}{c}\text { New } \\
\text { Industries }\end{array}$ & YHLO & IFA \\
\hline \multirow[t]{5}{*}{$\overline{\text { un }}$} & 90 & 70 & 75 & 70 & 85 & 75 & 75 \\
\hline & 60 & & 75 & 80 & 65 & 55 & 75 \\
\hline & 65 & & & 85 & 90 & 60 & 75 \\
\hline & 70 & & & & 75 & 55 & 75 \\
\hline & 70 & & & & & 70 & 75 \\
\hline \multirow{2}{*}{$s$} & 70 & & & & & & 75 \\
\hline & 65 & & & & & & \\
\hline
\end{tabular}

a, Positive rate of assay in 20 patients.

b, Coincidence rate of positive and negative in 20 patients.

Table 4. Spearman correlations coefficients in the six assays. 


\begin{tabular}{lcccccr}
\hline acturer & Euroimmun & Beier & Tarcine & Antubio & $\begin{array}{c}\text { New } \\
\text { Industries }\end{array}$ & YHLO \\
\hline nmun & 1 & 0.4937 & 0.4646 & 0.4971 & 0.4396 & 0.5352 \\
\hline & 1 & 0.6951 & 0.9857 & 0.6232 & 0.6412 \\
\hline $\mathbf{e}$ & & 1 & 0.6347 & $\mathbf{0 . 9 4 5 9}$ & 0.5415 \\
\hline & & & & 1 & 0.5415 & 0.6171 \\
\hline ries & & & & & & 0.4467 \\
\hline
\end{tabular}

Bold represent results $>0.9$

Figures 

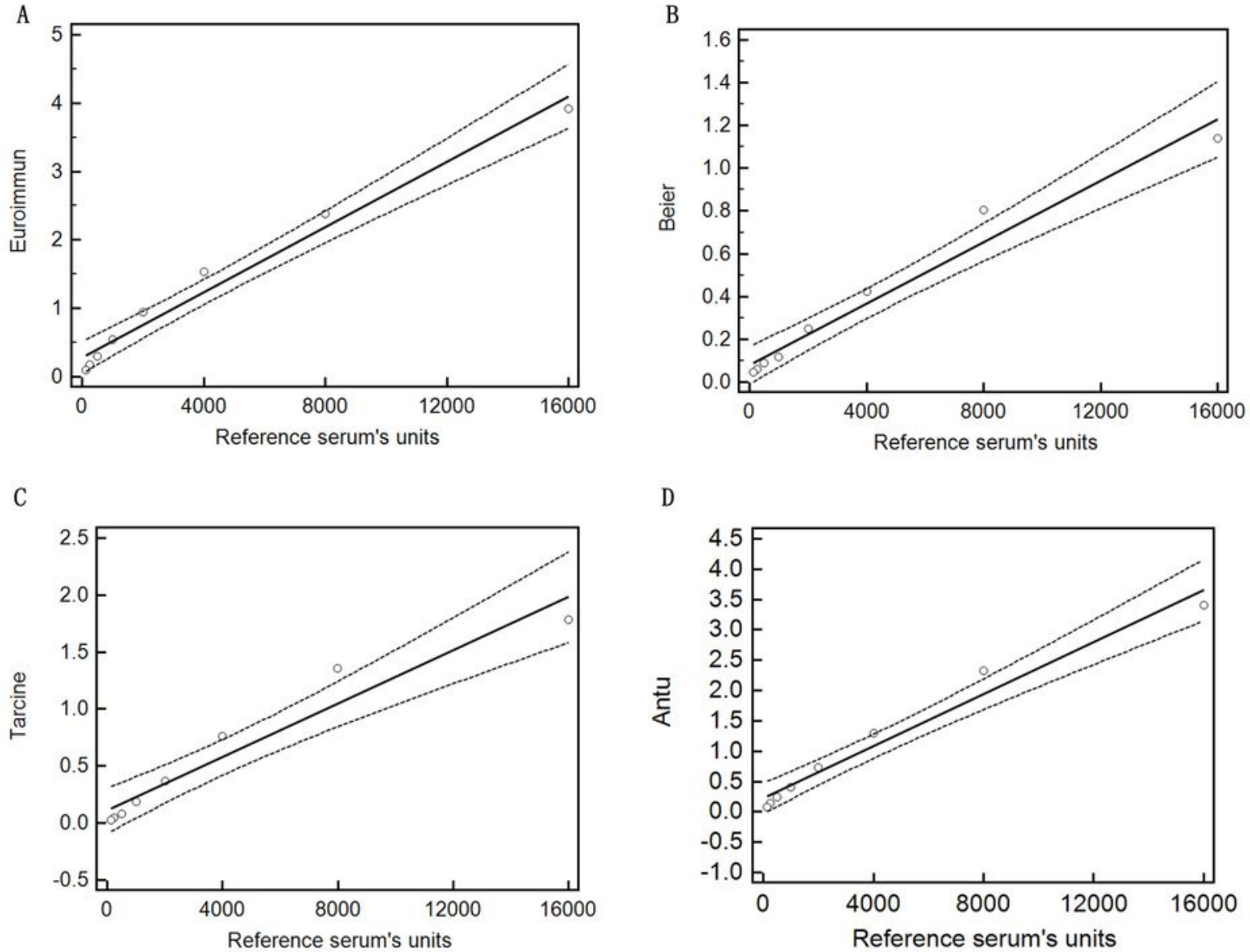

D

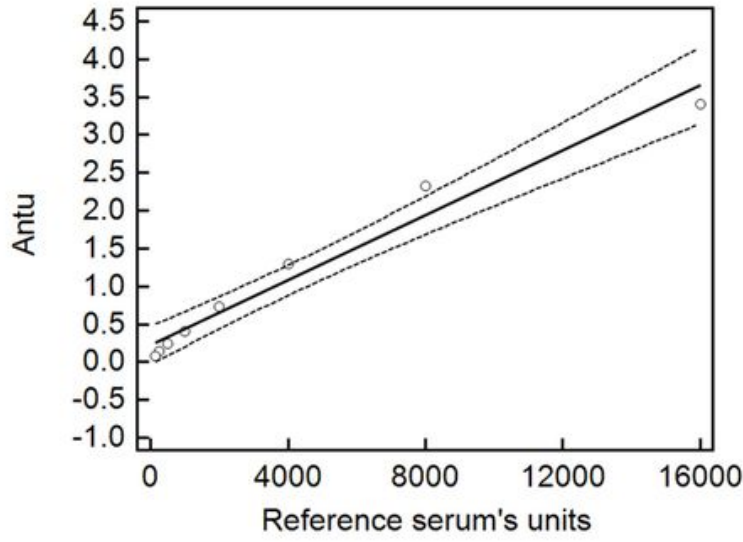

E
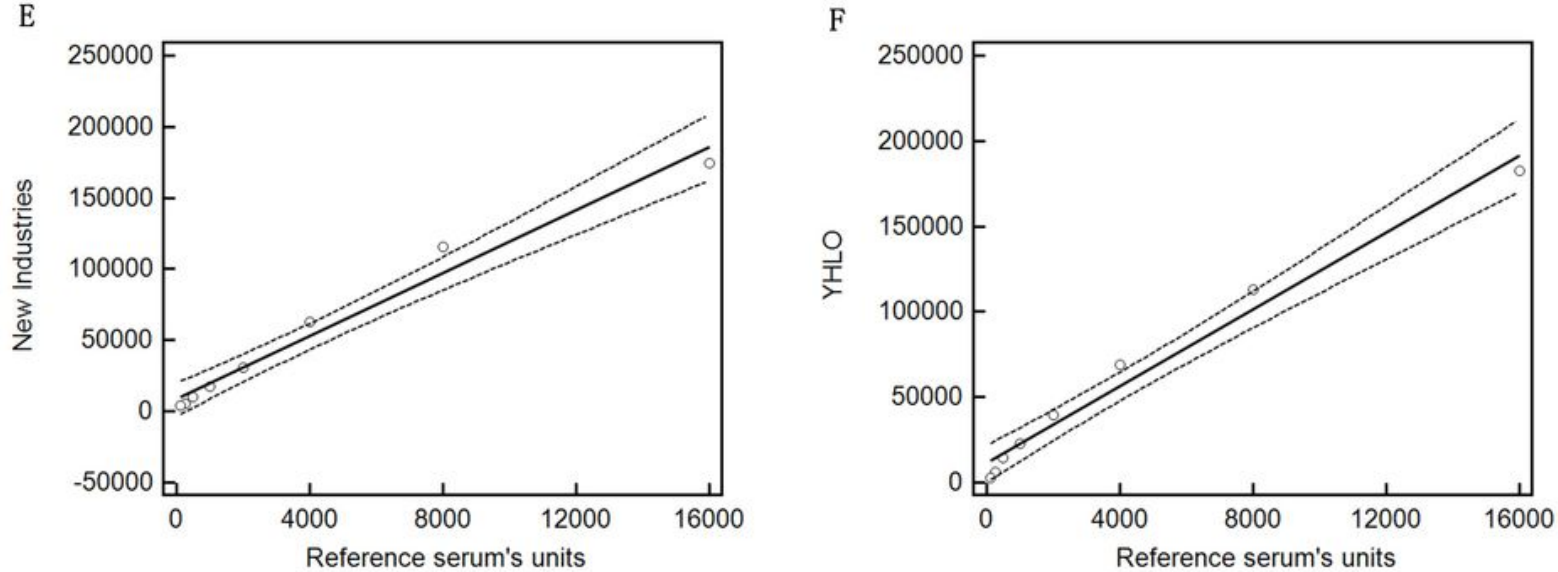

\section{Figure 1}

The regression curves obtained with each of the 6 commercial VCA-IgA methods. Values of the ordinate are given in the units used by the assay kit, while the abscissa shows the arbitrary units of the reference sample. 

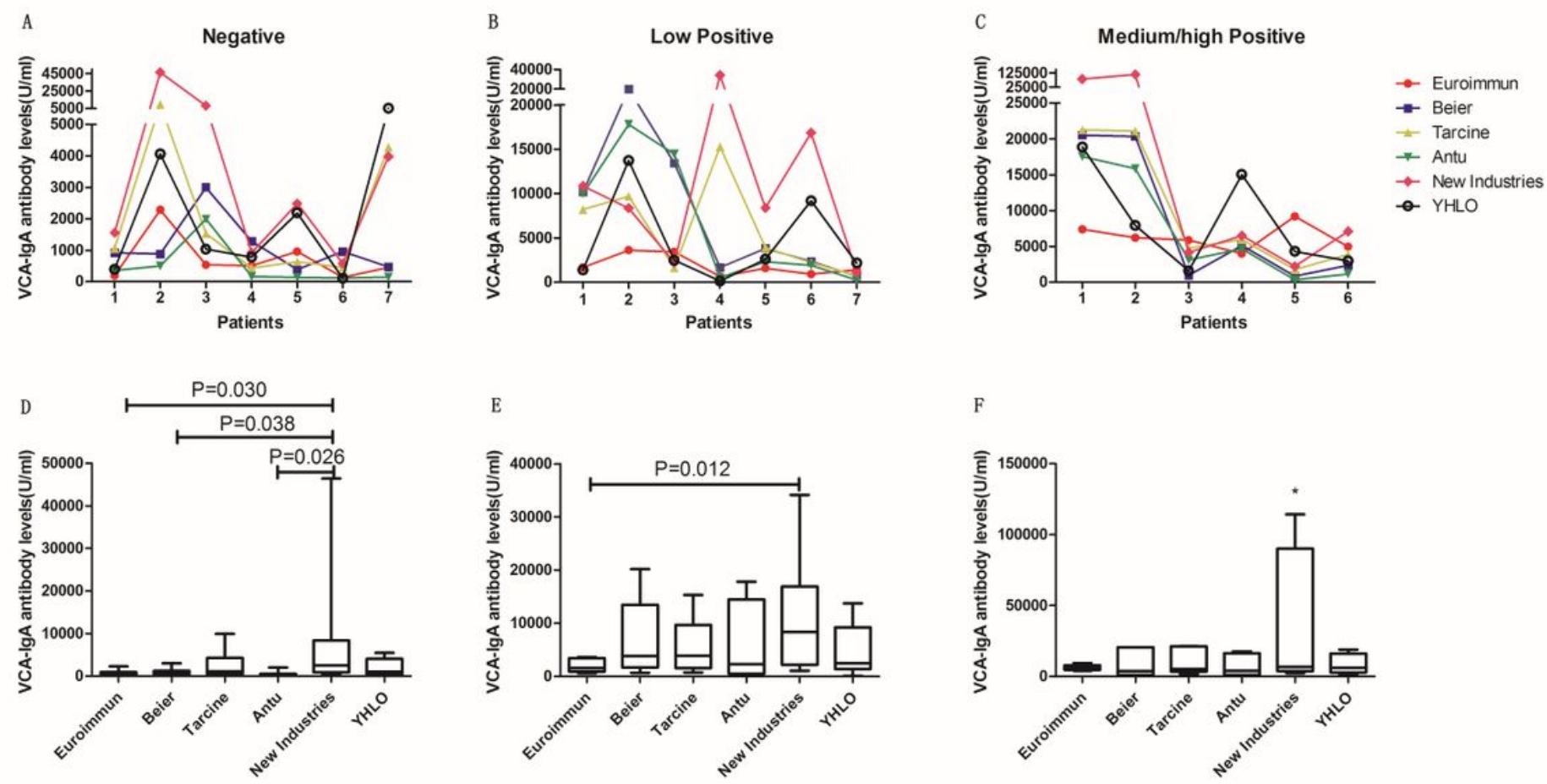

Figure 2

VCA-IgA results of 20 patients calibrated by the reference according three sample categories detected by 6 kits. Line chart of VCA-IgA results detected by each of 6 kits in three sample categories A, negative; B, low positive; $C$, medium/high positive. The mean VCA-IgA concentration of each patient by 6 methods divided into three sample categories were used LSD test to make multiple comparisons between the groups. D, negative;E, low positive; $F$, medium/high positive. It has been marked out, when the difference between the two groups is significant $(p<0.05)$. * New Industries group was different from other methods group in medium/high positive categories by LSD test $(P<0.05)$

\section{Supplementary Files}

This is a list of supplementary files associated with this preprint. Click to download.

- supplement1.docx

- supplement2.tif 